\title{
Delirium in older emergency department patients
}

\author{
Debra Eagles, MD, MSc* \\ INTRODUCTION
}

Delirium is common in older emergency department (ED) patients, with $10 \%$ prevalence in patients 65 years of age and older often cited. ${ }^{1}$ The sequelae of delirium are well described. Delirium is associated with increased hospitalization, increased likelihood of intensive care unit (ICU) admission, and increased length of stay. Patients with delirium often do not return to their premorbid functional or cognitive level and are at increased risk of institutionalization. They are seven times more likely to die than their non-delirious counterparts. With such high prevalence and devastating consequences, it is clear that delirium is a medical emergency. Yet, delirium is vastly underrecognized by ED healthcare professionals, going undiagnosed in $>65 \%$ of patients. ${ }^{2}$ The lack of delirium identification contributes to poor patient outcomes.

Recognizing this, in 2009, the Society for Academic Emergency Medicine Geriatric Task Force identified cognitive assessment as an area of ED care with significant quality care gaps. They recommended that the evaluation of all older patients include a screening cognitive assessment and, if found to be abnormal, further assessment to evaluate for delirium. ${ }^{3}$ This is reiterated in recent Geriatric ED Guidelines, a consensus document of the Society for Academic Emergency Medicine, the American College of Emergency Physicians, the American Geriatrics Society, and the Emergency Nurses Association, designed "to provide a standardized set of guidelines that can effectively improve the care of the geriatric population."4

One of the barriers in moving forward the agenda of cognitive evaluation of older people in the ED is the underlying knowledge gaps and the lack of clear gold standard practices in the recognition of delirium in the ED. As such, delirium has been identified as a key area in geriatric emergency medicine research, ${ }^{5}$ in which the development of delirium screening/diagnostic instruments is a top priority. Currently, the Diagnostic and Statistical Manual of Mental Disorders, 5th edition (DSM-V), criteria are considered the gold standard for delirium diagnosis; however, this entails a psychiatrist, neurologist, or geriatrician completing an in-depth neuro-cognitive evaluation. Clearly, this is not feasible within many acute care settings, particularly within the busy, dynamic environment of the ED. Thus, research has largely focused on the development of ED delirium screening tools with good diagnostic characteristics that are short, do not require additional tools and are easy to administer and interpret.

Although there has been a recent proliferation of research on delirium in the literature, there are still large knowledge gaps that warrant further investigation through studies with scientifically rigorous methodologies. The development of a delirium core outcome set that can be used across multiple healthcare settings will facilitate the standardized selection and reporting of delirium outcomes. ${ }^{6}$ Issues surrounding consent must be addressed, so that those at greatest risk of delirium who would most benefit from the research are appropriately included. The agreement of gold standard testing in the ED, time of first assessment, and time of re-assessment to differentiate between prevalent and incident ED delirium must be clarified.

Deriving the "best" delirium diagnostic instrument is the first step towards improved delirium care. Incorporating it into widespread practice is the only way to truly affect patient outcomes. To truly gauge performance in clinical settings, robust implementation studies based on established knowledge translation constructs are needed. The success of implementation is often predicated on identification and mitigation of barriers to behaviour change, as such a better understanding of patient, healthcare provider, and environmental/system factors that contribute to missed delirium must be elucidated. Although Kakuma et al. reported that ED identification of delirium alone decreased mortality, ${ }^{7}$ the question remains:

From the *Department of Emergency Medicine, University of Ottawa, Ottawa, ON.

Correspondence to: Dr. Debra Eagles, The Ottawa Hospital, Civic Campus, Clinical Epidemiology Program, Room F658, 1053 Carling Avenue, Box 685, Ottawa, ON K1Y 4E9; Email: deagles@toh.ca 
will widespread screening of older ED patients improve delirium identification and subsequent patient morbidity and mortality? Also, can a clinical decision rule be used to identify older patients with delirium who are safe to discharge from the ED? Research in delirium serum biomarkers, such as S-100 beta and IGLF-1, may provide clues to the underlying pathophysiology of delirium. More research is needed to clarify the utility of biomarkers in the diagnosis, prognostication, and monitoring of delirium. Ultimately, these biomarkers may serve as targets for treatment. Finally, there is evidence that preventive and management strategies in the ICU and hospital ward settings are effective; however, the applicability to the ED is unclear. Randomized controlled trials evaluating these and other novel approaches are warranted.

In this issue of CFEM, two articles addressing delirium in older ED patients are presented. The first reports on the performance of the French version of the 4AT: a four-question instrument designed to detect delirium. The 4AT was derived in an inpatient population but was recently validated in an ED population, with a reported sensitivity of $93 \%$ and specificity of $91 \% .^{8}$ Gagne et al. evaluated the use of the $4 \mathrm{AT}-\mathrm{F}$ as a screening tool for cognitive impairment in functionally semi-independent and independent patients age 65 years and older who were in the ED for a minimum of 8 hours, admitted or waiting to be admitted to the hospital. ${ }^{9}$ They reported a sensitivity of $84 \%$ (95\% confidence interval [CI]: 76-93) and a specificity of $74 \%$ (95\% CI: 70-78) when compared with the Confusion Assessment Method (CAM) administered by a research assistant. There are only two other tools, the Recognizing Acute Delirium As part of your Routine (RADAR) and the CAM-ICU, that have been reported in the French-speaking population; ${ }^{10}$ the 4AT-French is the only version that has been explicitly validated in a French-speaking, older, ED cohort.

In the second article, Thompson et al. examined risk factors associated with in-hospital delirium within 72 hours of ED presentation in older patients with hip fracture. ${ }^{11}$ They found age $>75$ years, history of dementia, and no analgesia were risk factors for delirium. The latter has important implications as a modifiable risk factor for delirium. While more study is needed, there is some evidence that regional anesthesia can reduce the risk of delirium. ${ }^{12} \mathrm{~A}$ better understanding of risk factors helps clinicians identify those at increased risk of delirium and researchers to evaluate targeted intervention strategies.

There remains much opportunity in delirium research. However, we do know that 1) delirium is common in older patients; 2) delirium is a significant burden to the patient and the healthcare system; 3) there are validated ED clinical tools that can screen for delirium; and 4) there is growing evidence that identification, prevention, and management strategies improve outcomes. Quality ED care in our older patients includes a cognitive evaluation. Will you do a cognitive evaluation on your next older ED patient to screen for delirium?

Keywords: delirium, emergency department, geriatrics

Acknowledgements: Thanks to Brittany Ellis MD, MSc, for providing feedback.

Competing interests: None declared.

\section{REFERENCES}

1. Elie M, Rousseau F, Cole M, et al. Prevalence and detection of delirium in elderly emergency department patients. CMA7 2000;163(8):977-81.

2. Han JH, Zimmerman EE, Cutler N, et al. Delirium in older emergency department patients: recognition, risk factors, and psychomotor subtypes. Acad Emerg Med 2009;16(3):193-200.

3. Terrell KM, Hustey FM, Hwang U, et al. Quality indicators for geriatric emergency care. Acad Emerg Med 2009;16(5):441-9.

4. The Geriatrics Emergency Department Guidelines. Ann Emerg Med 2014;63(5):e5.

5. Carpenter CR, Shah MN, Hustey FM, et al. High yield research opportunities in geriatric emergency medicine: prehospital care, delirium, adverse drug events, and falls. $\mathcal{7}$ Gerontol A Biolog Sci Med Sci 2011;66(7):775-83.

6. Rose L, Agar M, Burry LD, et al. Development of core outcome sets for effectiveness trials of interventions to prevent and/or treat delirium (Del-COrS): study protocol. BM7 Open 2017;7(9):e016371.

7. Kakuma R, du Fort GG, Arsenault L, et al. Delirium in older emergency department patients discharged home: effect on survival. 7 Am Geriatr Soc 2003;51(4):443-50.

8. O'Sullivan D, Brady N, Manning E, et al. Validation of the 6-item cognitive impairment test and the 4AT test for combined delirium and dementia screening in older emergency department attendees. Age Ageing 2018;47(1):61-8.

9. Gagné A-J, Voyer P, Boucher V, et al. Performance of the French version of the 4AT for screening the elderly for delirium in the emergency department. CFEM 2018;20(6):903-10.

10. Voyer P, Champoux N, Desrosiers J, et al. Recognizing acute delirium as part of your routine [RADAR]: a validation study. BMC Nurs 2015;14:19, doi:10.1186/s12912-015-0070-1.

11. Thompson C, Brienza VJM, Sandre A, et al. Risk factors associated with acute in-hospital delirium for patients diagnosed with a hip fracture in the emergency department. C7EM 2018;20(6):911-19.

12. Abou-Setta AM, Beaupre LA, Rashiq S, et al. Comparative effectiveness of pain management interventions for hip fracture: a systematic review. Ann Intern Med 2011;155(4):234-45. 